\title{
Naciones Unidas;
}

Posicionamiento y Debate Sobre La Orientación Sexual e Identidad de Género

\author{
Cristhian Manuel Jiménez \\ Graduado en Diplomacia o Servicios Internacionales \\ (2008) y especialista en Docencia Universitaria (2010) \\ por la Universidad Católica Santo Domingo (Rep. Domi- \\ nicana), magister en Gerencia de los Recursos Humanos \\ (2012) por la Universidad Apec (Rep. Dominicana) y \\ es becario OEA-GCUB 2014 de la Maestría en Ciencias \\ Humanas y Sociales (2015-20117) en la Universidade \\ Federal do $A B C$ (Brasil). Es vice-presidente y fundador \\ de la Fundación Comunidad Esperanza y Justicia Interna- \\ cional (Funceji). Profesor de la Universidad Dominicana \\ 0\&M (2012-2015).cejird@gmail.com
}

\section{Gilberto M. A. Rodrigues}

Profesor del Curso de Relaciones Internacionales y de Pos-grado en Ciencias Humanas y Sociales de la Universidade Federal do ABC. Investigador del CNPq. Pos-doctorado (Fulbright) por el Center for Civil and Human Rights, Universidade de Notre Dame (EUA). Fellow Research, CLALS, American University, Washington, DC. gilberto. rodrigues@ufabc.edu.br

\section{Resumen}

¿Cuáles son los principales argumentos que definen el debate sobre la Orientación Sexual e Identidad de Género en la ONU? ¿Cómo las Naciones Unidas se posicionan sobre la protección a las personas LGBTI? ¿Cómo los mecanismos de protección internacional han interpretado los pactos 
y convenciones para proteger a las personas LGBTI? Son estas las interrogantes que marcan este artículo, cuyo objetivo será analizar el desarrollo del debate entre los Estados sobre la Orientación Sexual e Identidad de Género y el posicionamiento de la organización multilateral de mayor relevancia en el mundo.

Palabras clave: LGBTI. ONU. Discriminación. Derechos humanos. Derecho Internacional de los Derechos Humanos.

\title{
United Nations; Stance and Debate on Sexual Orientation and Gender Identity
}

\begin{abstract}
What are the main arguments that define the debate on Sexual Orientation and Gender Identity at the UN? How the United Nations stance on protecting LGBTI people? How international protection mechanisms have interpreted the covenants and conventions to protect LGBTI people? These question marks this paper. The aim of this paper will be to analyze the development of the debate among States on Sexual Orientation and Gender Identity and the stance of the most important multilateral organization in the world.
\end{abstract}

Keywords: LGBTI. United Nations. Discrimination. Human Rights. International Human Rights.

\section{Sumário}

1 Introducción. 2 Debate Entre los Estados Sobre la Orientación Sexual e Identidad de Género (OSIG). 2.1 Comisión de Derechos Humanos. 2.2 Debates en la Asamblea General. 2.3 Consejo de Derechos Humanos. 2.4 Examen Periódico Universal (EPU). 2.5 Principios de Yogyakarta. 2.6 Consejo de Seguridad. 2.7 Principales argumentos que definen el debate. 3 Posicionamiento de la ONU Sobre la Protección a las Personas LGBTI. 3.1 Secretario General de las Naciones Unidas. 3.2 Oficina del Alto Comisionado de las Naciones Unidas para Ios Derechos Humanos (OACDH). 3.3 Agencias especializadas y Programas de la ONU. 4 Posicionamiento de los mecanismos de tratados y procedimientos especiales. 5 Conclusiones. 6 Referencias. 


\section{INTRODUCCIÓN}

Una de las minorías cuyos derechos a la igualdad, la libertad y dignidad sigue siendo cuestionado por grupos de poder son las personas Lesbianas, Gays, Trans, Bisexuales e Intersexuales (LGBTI). Según el reporte anual Homofobia de Estado 2016 de la Asociación Internacional de Lesbianas, Gays, Bisexuales, personas Trans e Intersex (International Lesbian and Gay Association - ILGA), 73 países del mundo criminalizan a las personas por su orientación sexual y pueden ser encarceladas por ser quienes son, la mayoría de estas leyes son remanentes del periodo colonial. A la vez el informe demuestra que las personas LGTBI son objeto de discriminación en el acceso a todos sus derechos alrededor del mundo y no gozan de protección y garantías contra la violencia, tortura, asesinatos y persecución. A penas unos 22 países aprueban el matrimonio de personas del mismo sexo, el cambio de identidad sexual y género, 26 permiten la adopción de niños y niñas por parejas del mismo sexo, 39 disponen de legislación antidiscriminación que incluye a las personas LGBTI, 40 países prohíben la incitación al odio (ILGA, 2016). Lo anterior demuestra que aunque varios países han realizado esfuerzos, aun en la mayoría de los países del mundo las vidas de las personas LGBTI son precarias.

Desde el nacimiento de la ONU, su carta fundadora declara en su artículo 1, párrafo 3 que uno de sus propósitos es `el desarrollo y estímulo del respeto a los derechos humanos y a las libertades fundamentales de todos" (NACIONES UNIDAS, 1945), así mismo en el artículo 55 párrafo c establece que promoverá el respeto universal a los derechos humanos y en el artículo 56 compromete a todos los miembros a tomar medidas para cumplir con este propósito. Esta obligación de los estados miembros de la ONU se refuerza con la Declaración Universal de los Derechos Humanos (DUDH) de 1948 y la Declaración y Programa de Acción de Viena de 1993. Como expone José Augusto Lindgren Alves "los derechos humanos ya no son materia de exclusiva competencia de las jurisdicciones nacionales. Su observancia es exigencia universal consensualmente acordado por los 
Estados” (ALVES, 2003, p. 41). Como menciona el mismo autor a partir de la DUDH "el hombre y la mujer pasan a convertirse en sujetos de derecho internacional, ocupando un lugar como ciudadanos del mundo, un espacio previamente reservado apenas a los Estados" (p. 37). Con esto queda claro que la ONU tiene un deber importante en la lucha contra la discriminación por cualquier condición, incluida la orientación sexual e identidad de género (en adelante OSIG). El enfoque en promover el reconocimiento LGBTI en el discurso de la ONU es importante debido a la fuerza de creación de normas del organismo.

Expuesto el panorama anterior y el deber de la ONU, surgen las preguntas sobre ¿Cuáles son los principales argumentos que definen el debate sobre la Orientación Sexual e Identidad de Género en la ONU? ¿Cómo las Naciones Unidas se posicionan sobre la protección a las personas LGBTI? ¿Cómo los mecanismos de protección internacional han interpretado los pactos y convenciones para proteger a las personas LGBTI? Son estas las interrogantes que marcan este artículo, cuyo objetivo será analizar el desarrollo del debate entre los Estados sobre la orientación sexual e identidad de género en la ONU y el posicionamiento de esta organización multilateral.

\section{DEBATE ENTRE LOS ESTADOS SOBRE LA ORIENTACIÓN SEXUAL E IDENTIDAD DE GÉNERO (OSIG)}

En 1983 una resolución del Consejo Económico y Social (ECOSOC) de la ONU recomendó la realización de una investigación sobre minorías sexuales al Centro de Derechos Humanos ${ }^{1}$. El informe fue presentado en

1 El Centro de Derechos Humanos era la oficina del secretariado encargada de la promoción y protección de los Derechos Humanos de todo el sistema ONU. Hoy en día es conocida como la Oficina del Alto Comisionado de las Naciones Unidas para los Derechos Humanos. 
1988 a la Subcomisión de prevención de la discriminación y las minorías, por Fernand-Laurent (SANDERS, 2002). El informe debía responder a si las minorías sexuales ${ }^{2}$ eran objeto de discriminación y si dicha discriminación era justa en cualquier situación. El informe expuso que ningún país estaba exento de cambios en la permisibilidad de conductas sexuales que riñen con la moral, pero que dicho deterioro podía verse impactada por un retorno a los valores tradicionales, lo que volvería a marginalizar a las minorías sexuales. El estudio pide que se eliminen las causas que provocan sufrimiento a las minorías sexuales y respeto para que encajen en sociedad. Sin embargo el estudio expone que los estereotipos de género que son impuestos en la sociedad, que obligan a los hombres a ser viriles y a las mujeres a ser femeninas, son las causas de las personas se conviertan en homosexuales ${ }^{3}$ y lesbianas. Ambas aseveraciones son homofóbicas y no parten de ninguna evidencia científica, lo que será comprobado en 1990 cuando la OMS decide despatologizar la homosexualidad. También el estudio solicito a la Comisión de Derechos Humanos ponderar que derechos merecen las minorías sexuales. Solicito que se adopten leyes que criminalicen la homofobia, el respeto por la privacidad de las relaciones sexuales entre adultos del mismo sexo, la operación de cambio de sexo como condición para realizar cambios en la identidad sexual y permitir la formación de asociaciones no gubernamentales a las minorías sexuales. Sin embargo el estudio finaliza exponiendo que los Estados tienen la obligación de proteger a las minorías sexuales, pero que antes debe ponderar la protección de la sociedad sobre prácticas que amenazan la moral, el orden público y el bienestar de la sociedad. Por lo que cuando un derecho entre en confrontación entre ambos grupos, el Estado debe favorecer la protección de los más vulnerables, que son la mujer y los niños. A la vez

2 El informe entendía las minorías sexuales como homosexuales, lesbianas y transexuales.

3 El estudio dice que si los hombres fueran más cariñosos con las mujeres hubieran menos lesbianas, y si a los hombres se les exigiera menos en su performance sexual, no hubiera tantas personas con problemas de impotencia u homosexuales. 
que pide que los Estados tomen las medidas necesarias para proteger la salud general de la población, por los riesgos para la salud que representan los homosexuales ${ }^{4}$. Este estudio como vemos aunque presenta algunas medidas rescatables, esta permeado por la homofobia y el heterosexismo de la época, donde las personas LGBTI eran vistas como enfermas, producto de un deterioro de la moral social y un riesgo para la población, y en especial para los niños (EDWARD H. LAWSON, 1996). El informe no causo ninguna empatía en la subcomisión ni se le dio seguimiento.

La Conferencia de Viena del 1993 fue la primera vez en que organizaciones gays y lesbianas fueron formalmente acreditadas en una conferencia de la ONU. Organizaciones feministas lograron que Canadá propusiera adherir en la lista de los tipos de discriminación la orientación sexual, sin embargo dicha propuesta no fue aprobada (GIRARD, 2004, p. 358). En 1994 los grupos feministas y LGTBI realizaron esfuerzos sin ningún éxito para insertar la no discriminación por orientación sexual y los derechos sexuales y reproductivos en los planes y declaraciones en la Conferencia Internacional sobre la Población y el Desarrollo del Cairo 1994 y la Conferencia Mundial sobre la Mujer en Beijing 1995, durante esta última 'Vanuatu, con apoyo de Canadá, solicitó una referencia a la discriminación con base en la orientación sexual, en el capítulo de la familia, pero esa referencia fue rechazada” (GIRARD, 2004, p. 362). Durante la Conferencia Mundial contra el Racismo, la Discriminación Racial, la Xenofobia y las Formas Conexas de Intolerancia en Durban en 2001, Brasil, Canadá, Chile, Ecuador y Guatemala (ORGANIZAÇÃO..., 2001) promovieron la inclusión de la orientación sexual en el programa de acción, sin embargo al igual que en las conferencias pasadas el Vaticano y sus aliados, así como

4 Se asume que el estudio se refiere a la epidemia del VIH-Sida que en ese momento estaba asociada solo a los homosexuales. 
la Confederación de Países Islámicos lograron bloquear la iniciativa, bajo los argumentos de que dichos términos eran una amenaza a la moral y las buenas costumbres de los países musulmanes y cristianos.

Tanto las experiencias de Viena, Cairo, Beijing y Durban sembraron el inicio de un debate que hoy ha ganado mayor campo gracias al movimiento feminista y LGTBI. También en estos primeros debates ya se escuchaban los argumentos que aun hoy en día retumban en las paredes de la ONU, ya que se sustenta que la discriminación por OSIGOSIG no está avalada por ningún instrumento internacional y que promoverlo sería atentar contra la soberanía de los países, olvidando la declaración de Viena respecto a que no se puede argüir al argumento de injerencia en términos de derechos humanos.

\subsection{Comisión de Derechos Humanos}

En 2003 la Delegación de Brasil en la extinta Comisión de Derechos Humanos sometió a discusión el proyecto de resolución E/ CN.4/2003/L.92 (INTERNATIONAL..., 2003) titulado "Los derechos humanos y la orientación sexual”. La resolución buscaba seguir lo iniciado en la conferencia mundial de Durban, de que los derechos humanos son aplicables a todas las personas sin importar su orientación sexual y que por lo tanto las personas no deben ser perseguidas, asesinadas, torturadas o encarceladas por esta razón. El proyecto de resolución conto con el apoyo de varios países. ${ }^{5} \mathrm{y}$ decenas de organizaciones no gubernamentales, sin embargo también levanto un frente opositor agresivo con el Vaticano y Pakistán a la cabeza. Por un lado el Vaticano sostuvo que aunque condena todo tipo de violencia incluida la ejercida contra personas homosexuales,

5 Alemania, Austria, Bélgica, Brasil, Canadá, Dinamarca, España, Finlandia, Francia, Grecia, Irlanda, Italia, Liechtenstein, Luxemburgo, Noruega, Países Bajos, Portugal, Reino Unido de Gran Bretaña e Irlanda del Norte, República Checa y Suecia. 
veía en la resolución una intención perversa de promover la homosexualidad. La primera respuesta de Pakistán fue que en nombre de la Organización de la Conferencia Islámica promovió una moción de no acción para sacar la resolución de la agenda, argumentando que dicha resolución crearía nuevos derechos que estaban en desacuerdo con los valores culturales y religiosos de muchos estados, y que ese tema no era propicio para la comisión, por lo que crearía más discordia, división o incluso algo peor. Brasil tomo la palabra y se lamentó por tal posición y realizo un llamado para votar en contra de dicha moción. Brasil logro una victoria breve al ganar la votación ${ }^{6}$ (ORGANIZAÇÃO..., 2003a). En su 63ª sesión, celebrada el 25 de abril de 2003, la Comisión de Derechos Humanos decidió 7 aplazar para 2004 el examen del proyecto de resolución E/CN.4/2003/L.92. Brasil perdió el apoyo de varios países como Argentina que paso a votar a favor del aplazamiento y otros que pasaron a abstenerse como fueron Armenia, Australia, Estados Unidos, Paraguay y Perú.

Ante lo sucedido en 2003 el movimiento LGTBI esperaba la nueva discusión de la resolución en 2004, sin embargo llegada la fecha Brasil anuncio que retiraría la resolución debido a que el asunto se politizo y había generado discordia entre países amigos. Girard menciona que "aparentemente se había ejercido una tremenda presión en Brasil por parte de los estados de la OCI (Organización de la Conferencia Islámica), la cual amenazaba con boicotear una cumbre de comercio de la cual Brasil sería el anfitrión” (GIRARD, 2004, p. 386).

La comisión aplazo el examen de la cuestión para 2005, en esta oportunidad Nueva Zelanda ${ }^{8}$ hizo una declaración, en la cual dijo que "no podemos ignorar el montón de evidencia de serias violaciones a los dere-

\footnotetext{
624 en contra, 22 a favor y 6 abstenciones.

7 Por votación registrada de 24 votos contra 17 y 10 abstenciones.

8 La declaración conto con el apoyo de 32 países.
} 
chos humanos en contra de individuos por su orientación sexual, estas violaciones han llamado nuestra atención y debemos responder (ARC INTERNATIONAL, 2005).

Según Beto de Jesús (JESUS, 2004) la propuesta brasileña fue presentada sin antes conversar con los países que ya trabajaban el tema en la ONU y el movimiento LGBTI, Brasil no había anticipado el rechazo que tuvo la resolución, y los discursos que acusaban de pedofilia promover dicho tema. Ningún país latinoamericano copatrocino la resolución. Ante la resistencia de Brasil de seguir con la resolución, también salió de relieve la necesidad de la búsqueda de un líder de dicha iniciativa que fuese del sur y no del norte debido a que se vería como una imposición de los países europeos, lo que termino con la declaración de Nueva Zelanda. Sin embargo la iniciativa Brasileña sirvió para movilizar y poner en la agenda de debate en la ONU la orientación sexual, lo que promovería una mayor acción del movimiento internacional LGBTI así como de los Estados en diferentes órganos políticos de las Naciones Unidas a través de los años.

\subsection{Debates en la Asamblea General}

Desde el año 2000 en el tercer comité de la Asamblea General bajo el liderazgo de Suecia y otros países nórdicos se ha logrado aprobar cada dos años (2000, 2002, 2004, 2008, 2010, ${ }^{9} 2012$ y 2014) la inclusión de la protección del derecho a la vida de las personas LGTBI y la debida investigación cuando fuesen víctimas en la resolución sobre Ejecuciones extra-

9 Durante la discusión del 2010 en el tercer comité de la Asamblea General el grupo árabe, el grupo africano, los países de la OCI y algunos países caribeños presentaron una enmienda para retirar la palabra "orientación sexual" de la resolución. La enmienda fue aprobada con 79 votos a favor, 70 en contra y 17 abstenciones. Sin embargo Suecia y sus aliados consiguieron incluir nuevamente la palabra cuando el proyecto de resolución llego al plenario de la Asamblea General, en esa ocasión fue aprobada por 93 votos contra 55 en contra y 27 abstenciones. 
judiciales, sumarias o arbitrarias. Durante las discusiones del 2002, para adoptar el informe del Relator de Ejecuciones Extrajudiciales, 44 países entre ellos Sudan, Irán, Arabia Saudita, Malasia, Líbano y Pakistán intentaron mediante una enmienda sacar de la resolución la mención respecto a la orientación sexual así como argumentar que las ejecuciones no las cometen los gobiernos sino civiles ajenos a la autoridad. El representante del Líbano dijo que; 'Incluir una sección completa al asunto de la orientación sexual y las minorías sexuales implica un endoso de una categoría particular a otra. El representante dijo que algunos países tienen reservas debido a razones religiosas y culturales respecto a dicha referencia" (ORGANIZAÇÃO..., 2002).

La enmienda no pasó ${ }^{10}$. Finlandia y demás países que se opusieron a la enmienda fueron muy claros al explicar que todas las muertes deben investigarse, incluso las de personas con una orientación sexual diferente a la heterosexual (ORGANIZAÇÃO..., 2002). A partir de 2012 fue incluido el término de identidad de género en la resolución. Esta resolución es de gran importancia debido a que la Asamblea General es el órgano de mayor importancia y alcance de la ONU debido a que está compuesta por todos los países miembros (RODRIGUES, 2014), quienes han aprobado en repetidas ocasiones esta resolución, lo que le da fuerza internacional al derecho de no ser objeto de violencia en razón de la OSIG.

Otra iniciativa dentro de la Asamblea General que provoco un amplio debate sucedió en diciembre de 2008, cuando bajo la coordinación de países de los cuatro continentes; Argentina, Brasil, Croacia, Francia, Gabón, Japón, Noruega y Países Bajos se presentó por primera vez ante la Asamblea General de las Naciones Unidas una declaración abordando las violaciones de derechos humanos basadas en la OSIG, la declaración conto

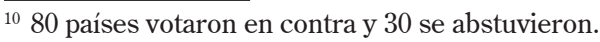


con el respaldo inicial de 66 países. ${ }^{11}$ Dicha declaración fue propuesta por Francia como presidente de la Unión Europea y fue leída por Argentina quien en 2003 tenía una posición en contra del tema al votar a favor del aplazamiento de la resolución promovida por Brasil, también Gabón voto en esa oportunidad a favor en la comisión de derechos humanos. La declaración reafirmo el principio de universalidad y no discriminación incluyendo la orientación sexual, realiza un llamado a la despenalización de la homosexualidad, a proteger a los defensores de derechos humanos LGTBI, a condenar la violencia y discriminación y garantizar cero impunidad y una investigación efectiva de los hechos. Pide al Consejo de Derechos Humanos realizar una discusión sobre el tema y promueve al sistema universal de la ONU a promocionar los derechos de las personas LGTBI. Ante esta declaración inmediatamente el Vaticano pidió la palabra e intervino apoyando la no discriminación y violencia contra las personas homosexuales sin embargo dijo que dicha declaración se proponía otros fines mayores a la condena y expreso que 'En particular, las categorías “orientación sexual” e “identidad de género”, empleadas en el texto, no encuentran reconocimiento o una definición clara y compartida en el derecho internacional” (VATICANO, 2008). A la vez Siria promovió al día siguiente una contra declaración firmada por 59 países, ${ }^{12}$ entre los países firmantes sale a relucir que Santa Lucia y Guyana ${ }^{13}$ fueron los únicos países miembros de la Organización de Estados Americanos (OEA) que firmaron la propuesta siria, habiendo aprobado este organismo por unanimidad en el mismo año una resolución similar a la declaración francesa ${ }^{14}$.

${ }^{11}$ Todos los países miembros de la Unión Europea y una gran parte de la OEA.

${ }^{12}$ La mayoría países musulmanes de áfrica y oriente medio.

${ }^{13}$ Ambos países criminalizan el sexo consensual entre adultos del mismo sexo.

${ }^{14} \mathrm{La}$ OEA ha aprobado por unanimidad resoluciones en contra de la violencia y discriminación por orientación sexual en 2008, 2009, 2010, 2011, 2012, 2013, 2014 y 2015. Que dichos países del caribe hayan votado en contra, representa una inconsistente política exterior sobre el asunto. 
Siria planteo que el derecho internacional no protege la no discriminación por orientación sexual y que la declaración francesa era un atentado a derecho interno de los países y a los principios de la carta de las Naciones Unidas sobre no injerencia y respeto de la soberanía, dice que promover la normalización de la homosexualidad daría cabida a otros comportamientos deplorables como la pedofilia, Siria plantea que no se pueden crear nuevos derechos más de los que fueron codificados en la Declaración Universal de Derechos Humanos, incluso argumenta que el artículo 29 de dicha declaración les permite ejecutar leyes para mantener la moral y el orden público. Siria culmina mandando a los estados a proteger el significado de la familia igual como lo expuso el Vaticano (PERMANENT..., 2008).

\subsection{Consejo de Derechos Humanos}

El 1 de diciembre de 2006 la delegación de Noruega ante el consejo de derechos humanos presento una declaración ${ }^{15}$ que fue elogiada por ser la primera vez que se incluye la identidad de género en un debate en la ONU. En marzo de 2011, 85 estados presentaron una declaración similar a la de 2006 y a la presentada en la Asamblea General en 2008. Esta declaración conto con un fuerte apoyo diplomático de los Estados Unidos y se logró obtener 20 países más que no se habían suscrito a ninguna de las anteriores declaraciones. Esta declaración sirvió para emprender el trabajo para la aprobación de una resolución en el consejo de derechos humanos, en el mismo año.

El Consejo de Derechos Humanos fue el primer órgano político en aprobar en 2011 y 2014 resoluciones a favor de la no discriminación por OSIG. La resolución ${ }^{16}$ del 2011 (CONSEJO..., 2011) logro cuatro grandes

\footnotetext{
${ }^{15}$ La declaración contó con el apoyo de 54 países de entre ellos 18 miembros del consejo de derechos humanos.

${ }^{16}$ Fue aprobada con 23 votos a favor, contra 19 y 3 abstenciones.
} 
avances; el primero fue la realización de un estudio que documento las legislaciones y prácticas de discriminación y violencia cometidos contra personas LGTBI realizado por la Alta Comisionada de las Naciones Unidas para los Derechos Humanos y que incluyera recomendaciones dirigidas a los Estados para fortalecer la protección de los derechos de las personas LGTBI. El segundo avance fue la celebración de un panel el 7 de marzo de 2012 que tuvo como fundamentos el informe de la Alta Comisionada, esta fue la primera vez que un órgano intergubernamental de la ONU celebraba una actividad de debate sobre este asunto, sin embargo los países árabes y africanos como señal de protesta abandonaron el salón. El tercer avance es que la resolución se basa en que todos los individuos son sujetos de la universalidad de derechos, logrando así que el principio de universalidad de los derechos humanos sea extendido a las personas LGBTI con el apoyo de la comunidad internacional. Por último el cuarto avance fue el incluir la expresión de preocupación del consejo de derechos humanos hacia los actos violentos y la discriminación a personas LGBTI, a la vez que rompió el largo silencio en la ONU sobre las personas LGBTI.

La resolución de 2014 (CONSEJO..., 2014) fue similar a la anterior y solicito la actualización del informe. La resolución fue aprobada con 25 votos a favor contra 14 y 7 abstenciones. En ambas resoluciones Estados Unidos de América voto a favor, a diferencia de las abstenciones que registro en otras declaraciones anteriores, inicio una campaña a favor del reconocimiento y ha dispuesto todos los recursos de su departamento de Estado para promover la causa LGBTI en la ONU, pero trasladando el protagonismo a países aliados del sur global. Mientras Rusia quien se había abstenido también paso a votar en contra de las resoluciones, transformarse en un líder de la oposición y liberar presión del Vaticano.

En 2014 Egipto y Sierra Leona (ORGANIZAÇÃO..., 2014a) propusieron en el Consejo de Derechos Humanos una resolución para celebrar un panel sobre la protección de la familia, debido a que la resolucion 
no incluia dento del concepto de familia, otras formas de organización como aquellas formadas por parejas del mismo sexo Uruguay propuso una enmienda para agregar el concepto de "diversas formas de familia" (ORGANIZAÇÃO..., 2014b), dicha propuesta no paso y el proyecto fue aprobado. Debido al discurso sobre la protección de la familia vista solo como aquella entre sexos opuestos que mantienen los países del grupo africano, árabe, islámico, Rusia y otros con fuerte presión del Vaticano se pondero que dicha resolución y la mesa podría significar un riesgo en el avance del reconocimiento de los derechos de las personas LGTBI y sus familias.

En 2016 se adopta ${ }^{17}$ la tercera resolución sobre "protección en contra de violencia y discriminación basada en orientación sexual e identidad de género" (ORGANIZAÇÃO..., 2016), esta resolución fue presentada por Argentina, Brasil, Chile, Colombia, Costa Rica, México y Uruguay. ${ }^{18} \mathrm{La}$ resolución crea un Experto Independiente sobre Violencia y Discriminación basada en OSIG. Cuyo mandato tendrá una duración de 3 años. Este mecanismo servirá como un punto focal para combatir los desafíos que enfrentan las personas LGBTI a nivel global. Deberá presentar informes anuales al Consejo de Derechos Humanos y a la Asamblea General.

El debate que se generó en el Consejo de Derechos Humanos para aprobar la resolución fue intenso. Los países miembros de la Organización de Cooperación Islámica y Rusia fueron los que mantuvieron la oposición. Mientras que México, Suiza, Países Bajos, Reino Unido, Panamá, Eslovenia y Alemania tomaron la palabra para defender el proyecto. Los países contrarios a la resolución en primera instancia pusieron a votación una moción de no acción como lo hicieron en la resolución de Brasil de 2003 para evitar que el tema fuese tratado, sin embargo esto no fue aprobado.

\footnotetext{
${ }^{17}$ La resolución fue adoptada por 23 votos a favor, 18 en contra y 6 abstenciones. ${ }^{18}$ El documento también contó con la adhesión de 41 países.
} 
A seguidas estos mismos estados promovieron enmiendas para degradar el proyecto y lograron aprobar 7 . Dichas enmiendas en resumen incluyen dentro de las clausulas pre ambulares de la resolución los argumentos de que el reconocimiento de la OSIG por la ONU podría herir la sensibilidad de los valores regionales, culturales y religiosos de los países, así como inmiscuirse en debates domésticos sobre estos valores a nivel nacional. También se aprobó una enmienda que condena el uso de presiones externas y coercitivas por Estados desarrollados en procesos de decisión a nivel nacional sobre el reconocimiento o no de los derechos de las personas LGBTI. Otra enmienda se basa en que promover el reconocimiento de la OSIG deteriora el sistema internacional de derechos humanos, ya que estas categorías no están protegidas por el derecho internacional. También se aprobó una enmienda que protege la soberanía de los Estados respecto a sus leyes, valores religiosos, culturales y sociales sobre la aplicación de la resolución, es decir sobre la labor que llevara a cabo el Experto Independiente (ORGANIZAÇÃO..., 2016).

Las enmiendas aprobadas hicieron un gran daño a la resolución, ya que ataca justamente el principio de universalidad de los derechos humanos, sin oponer la soberanía, la cultura, la religión y la política como objeciones para garantizar las libertades fundamentales. Es de saberse que en próximos debates los países que promovieron dichas enmiendas las utilizaran para legitimar su discurso. Sin embargo aunque el propósito era volver el documento uno que no fuese aprobable ya que se tergiverso gran parte de su objeto, los países que propusieron el proyecto entendieron que ese era el precio a pagar para lograr crear el experto independiente.

En la votación de la resolución, hubo dos cambios de posiciones en países que habían apoyado en 2014. Uno fue el de Filipinas quien había votado a favor en 2014 y se abstuvo en esta ocasión, señalando que el Experto podría amenazar la soberanía de los Estados. El otro fue el de Sudáfrica quien había sido el promotor de la primera resolución aprobada 
en $2011^{19}$ y también se abstuvo en la votación, debido a que este país considera que no era el tiempo para dar ese paso y que se necesitaba un mayor diálogo sobre el asunto y una aproximación más horizontal con los países que aún mantienen legislaciones en contra de las personas LGBTI, el delegado dijo que la iniciativa era "arrogante y temeraria" (ORGANIZAÇÃO..., 2016).

A la vez varios Estados señalaron al final de la sesión que no cooperaran con el nuevo experto, por lo que dicho mecanismo será desafiado en su actuar y seguramente será confrontado cada vez que se refiera a uno de esos Estados, así como se vivirán caldeados debates cuando presente sus informes ante la Asamblea General y el Consejo de Derechos Humanos. Un ejemplo fue Rusia quien llamo al experto una iniciativa que no contribuiría, sino que causaría el fin del dialogo en el Consejo de Derechos Humanos y un desperdicio de fondos para la ONU. También veremos si al finalizar su periodo de tres años será mantenido o eliminado. El 30 de septiembre de 2016 fue elegido para el puesto Vitit Muntarbhorn. Muntarbhorn es un jurista y académico tailandés que ha sido en dos ocasiones relator de la ONU y miembro actual de la comisión de investigación en Siria. Muntarbhorn es un reconocido colaborador del movimiento LGBTI en Asia y colaboro en la elaboración de los principios de Yogyakarta. El mandato del experto fue motivo de un crecimiento en la polarización en el Consejo de Derechos Humanos en torno al tema de la OSIG, los países contrarios a la adopción de la resolución intentaron promover en el tercer comité, el quinto comité y la plenaria de la Asamblea General entre noviembre y diciembre de 2016 una resolución para parar el comienzo de los trabajos del experto hasta tanto se discutieran los fundamentos del derecho internacional sobre la OSIG en lo que se basaría el experto y que aún no contaban con un consenso internacional, sin embargo luego de

${ }^{19}$ Esta actitud de Sudáfrica también se debe a que la resolución de 2011 que fue patrocinada por Estado causo que dicho país enfrentara problemas con sus amigos y aliados de África. 
cuatro votaciones, el experto pudo comenzar sus trabajos ${ }^{20}$. Al finalizar los 3 años de mandato del mecanismo surgirán preguntas como; ¿Cuál ha sido su impacto? ¿Cómo supero los obstáculos que hemos visto que enfrentara? y ¿Cómo incluyo dentro de su trabajo la agenda de la sociedad civil?

La derrota de Rusia como miembro del Consejo de Derechos Humanos en 2016 presenta una oportunidad para avanzar en la presentación de resoluciones en este foro, ya que Rusia es la única potencia que actúa como líder del bloque que rechaza las resoluciones sobre orientación sexual e identidad de género en la ONU. Sin embargo también la elección de Donald Trump como presidente de los Estados Unidos podría significar un cambio en la política exterior de ese país entorno a la cuestión de los derechos humanos de las personas LGBTI. Si los Estados Unidos pasa a una posición neutra como en antiguas administraciones republicanas o a una posición en contra podría estancar o incluso perjudicar al proceso de reconocimiento a nivel de Naciones Unidas de los derechos humanos de las personas LGBTI, debido a que la política exterior desplegada por el expresidente Barack Obama fue de gran relevancia para la aprobación de resoluciones en la ONU.

\subsection{Examen Periódico Universal (EPU)}

El Examen Periódico Universal (EPU) que se basa en un dialogo constructivo entre los miembros de la ONU respecto a los exámenes individuales de cada país sobre la situación en derechos humanos, ha sido un espacio favorable para avanzar en la descriminalización de la sodomía en

\footnotetext{
${ }^{20}$ Mayor información sobre el histórico de votaciones puede encontrarse en:

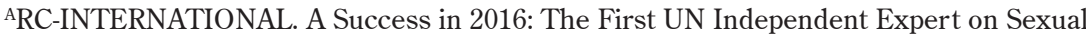
Orientation and Gender Identity. Disponible en: $<\mathrm{http}$ ://arc-international.net/research-and-publications/new-arc-reports/a-success-in-2016-the-first-un-independent-expert-on-sexual-orientation-and-gender-identity/>. Acceso en: 28 feb. 2017.
} 
diferentes países. Seychelles es un ejemplo reciente de un país que modifico su legislación luego de recibir recomendaciones en el EPU respecto al tema (SEYCHELLES NEWS AGENCY, 2016).

\subsection{Principios de Yogyakarta}

Debido a que ningún tratado internacional incluye las categorías de OSIG, así como el desarrollo dado de estas categorías recae en decisiones y comentarios generales de mecanismos de tratados, todos instrumentos del "soft law", el cual no es vinculante a los Estados miembros de la ONU, se hizo necesaria la elaboración de los principios de Yogyakarta. Estos principios son el esfuerzo de un conjunto de académicos y juristas por definir como se aplica el derecho internacional de los derechos humanos a las categorías de OSIG. Los principios fueron publicados en 2007 y han sido difundidos por todo el sistema de la ONU y el mundo. Hoy se consideran como el único y más importante documento internacional sobre la OSIG en el derecho internacional, por lo que varios países lo utilizan en sus discursos, así como lo han hecho oficiales de la ONU.

\subsection{Consejo de Seguridad}

Recientemente se ha llevado por primera vez a la atención del Consejo de Seguridad el tema de la no violencia contra personas LGTBI. En agosto de 2015 Chile y Estados Unidos invitaron a todos los miembros de las Naciones Unidas a una conversación a puerta cerrada con refugiados de países donde el Estado Islámico tiene control de algunos territorios y persigue y ejecuta a personas LGTBI, a la reunión no asistieron dos miembros no permanentes del Consejo. ${ }^{21}$

${ }^{21}$ Chad y Angola. 


\subsection{Principales argumentos que definen el debate}

Según la trayectoria del debate podemos distinguir dos bloques que se confrontan en la ONU. Por un lado un bloque de países que se manifiestan en contra del reconocimiento internacional de los derechos humanos de las personas LGBTI sostiene como argumentos lo siguiente; 1) Los conceptos de OSIG no tienen ninguna fundamentación en el derecho internacional al no estar establecidos explícitamente en ningún tratado internacional; 2) La intención de insertar nuevas categorías en instrumentos como la Declaración Universal de Derechos Humanos, es una malinterpretación sobre conceptos nunca previsto y una amenaza al sistema internacional de derechos humanos; 3) Dedicar tiempo al asunto va en detrimento de otros temas que son de prioridad como el racismo y la intolerancia religiosa; 4) Las conductas, estilos de vida y preferencias personales son asuntos de índole privado y no público, por lo que no son asuntos de derechos humanos y no necesitan ningún sistema de particular de protección. La homosexualidad es inaceptable para muchos países, ya que entra en contradicción con las enseñanzas de varias religiones, la cultura y los valores tradicionales de dichas sociedades, quienes promueven el debate sobre la OSIG no respetan la cultura y las particularidades de cada país. Dicho tema solo polariza el trabajo de la ONU y debe ser evitado; 5) La universalidad de los derechos humanos no permite la imposición de conceptos o ideas sin consenso y que van en contra de las especificidades de un conglomerado de países como es el caso de la OSIG. El principio de universalidad de los derechos humanos no debe ser utilizado para interferir en los asuntos de Estados soberanos que comprometen la legislación nacional o contradicen leyes divinas. Los Estados son libres para legislar por el bien de pueblo y ese derecho no puede ser limitado por la ONU o ningún otro Estado para imponer practicas ofensivas. 
Por su parte el bloque de países que procura el reconocimiento internacional de los derechos humanos de las personas LGBTI, plantea los siguientes argumentos y contestaciones a los expuestos por el bloque contrario; 1) Los derechos humanos de las personas LGBTI no son nuevas derechos, son los mismos derechos humanos ya dispuestos por los instrumentos internacionales y estos deben aplicarse a las personas LGBTI. Por lo que las leyes contra la sodomía, la censura a la libre expresión de la OSIG, que prohíben la libertad de reunión y otras similares, violan el derecho internacional de los derechos humanos; 2) Según la Declaración de Viena, la cultura, la religión y los valores tradicionales no pueden ser invocados para justificar la violencia y la discriminación a las personas LGBTI. Asegurar los derechos humanos de todas las personas requiere en ocasiones promover cambios en la cultura de los países. La libertad religiosa y la libertad de expresión no son amenazadas por respetar a las personas LGBTI. Podemos creer y decir lo que deseemos, pero no hacer o incentivar actos violentos y discriminatorios;3) Los redactores de los tratados de derechos humanos no excluyeron ningún individuo en el disfrute de las libertades fundamentales. Dentro del derecho internacional de los derechos humanos no existe ninguna cláusula de exclusión que justifique la violencia y discriminación a las personas LGBT. Por lo que los Estados no tienen derecho a proteger los derechos humanos de forma selectiva o excluir a las personas LGBTI de protección; 4) Aunque no se encuentre los conceptos de OSIG como categorías a ser no discriminadas en la declaración universal de derechos humanos, tampoco lo eran otros grupos vulnerables como las personas con discapacidad al momento, lo que quiere decir que la declaración y otros tratados internacionales de derechos no son restrictivos sino que son abiertos para incorporar diferentes grupos que sufran injusticia social; 5) La homosexualidad no es un fenómeno ocasionado por la decadencia del Occidente, es un rasgo de la conducta humana que ha existido en la historia de todas las sociedades del mundo, negar ese hecho es una excusa que no tiene ninguna validez; 6) 
Catalogar a las personas LGBTI como enfermas o posibles pedófilos son el resultado de mentiras y miedos sin ninguna base científica, utilizados para promover prejuicios contra las personas LGBTI; 7) Es responsabilidad de la comunidad internacional tratar abiertamente cualquier situación que amenace los derechos humanos en el mundo, no abordar la violencia y discriminación por OSIG implicaría ignorar el sufrimiento de millones de personas; 8) La violencia y la discriminación por OSIG no son asuntos de carácter privado; son cuestiones de interés público que amenazan a la sociedad en su conjunto; 9) No es necesario esperar que haya un consenso mundial común entorno a la OSIG para proteger los derechos humanos de las personas LGBTI.

Como se puede apreciar el primer bloque se fundamenta en el relativismo cultural, respeto a la soberanía, homofobia y una retórica anti occidente. El segundo bloque se concentra en la defensa de la universalidad de los derechos humanos, la garantía de la dignidad humana y la libertad de las personas LGBTI. Por lo que nos encontramos ante una polarización entre dos bloques que han adoptado posicionamientos que entran en conflicto.

\section{POSICIONAMIENTO DE LA ONU SOBRE LA PROTECCIÓN A LAS PERSONAS LGBTI}

\subsection{Secretario General de las Naciones Unidas}

En 2003 por primera vez el Secretario General de la ONU en su momento Kofi Annan se refirió a los derechos de las personas LGTBI, lo hizo en el marco de un acto que abordó el tema de la protección de los gays y lesbianas alrededor del mundo organizado por la asociación de empleados LGBTI de la ONU. Annan expreso que "las Naciones Unidas no pueden condonar ninguna persecución o discriminación en contra de personas bajo ningún caso, así como lo establece el artículo 2 de la Decla- 
ración Universal de los Derechos Humanos (ORGANIZAÇÃO..., 2003b). Sin embargo Annan agrego también que el tema había causado tensiones entre los estados miembros de las Naciones Unidas por lo que entendió que ese organismo no debería envolverse en ese asunto. Según un informe de Ilga Annan aprobó en 2004 extender los mismos beneficios a las parejas de empleados del mismo sexo que se hubiesen casado y su país aprobara dicho matrimonio serian reconocidas por la organización, el Vaticano y numerosas delegaciones de países islámicos y africanos presionaron en la Asamblea General para que el secretario reconsiderara, la misma fue revisada y se omitió la frase "pareja domestica" (SANDERS, 2002). En 2014 Ban anuncio que la igualdad debe iniciar por casa, con esto el secretario general a través de una orden administrativa aprobó reconocer todas las uniones de personas del mismo sexo siempre y cuando se hayan celebrado legalmente en un país donde se permita el matrimonio igualitario sin importar este fuese el de su nacionalidad como era la anterior condición expuesta por Annan. Los países tradicionalmente opuestos iniciaron a moverse para impedir el avance de esta iniciativa, Rusia tomo el liderazgo y propuso en marzo de 2015 en la quinta comisión de la Asamblea General vetar el alcance de los beneficios que el secretario pretendía dar a las parejas del mismo sexo, sin embargo el proyecto de resolución no paso por amplio margen con 43 votos a favor, 83 en contra y 37 abstenciones (REUTERS, 2015). También en 2008 Ban aprobó el reglamento anti discriminación del personal de la ONU, en el cual incluye la discriminación por orientación sexual (ORGANIZAÇÃO..., 2008).

Ban ha sido constante en su apoyo a las personas LGBTI en discursos y declaraciones ante la prensa, el Secretario General esta consiente de que aquellos que se oponen a través de argumentos sobre las tradiciones culturales y la religión, y ha expresado que dichos argumentos también han servido para justificar la esclavitud, la mutilación genital femenina y 
el matrimonio infantil. Ban entiende que aunque la religión y la tradición deben ser respetadas, estas nunca deben imponerse para negar los derechos humanos (KI-MOON, 2015).

\subsection{Oficina del Alto Comisionado de las Naciones Unidas para los Derechos Humanos (OACDH)}

La OACDH ha sido la agencia que ha jugado el rol más importante respecto a la lucha desde esta organización a la no discriminación por OSIG. Mary Robinson, Louise Arbour ${ }^{22}$ y Navi Pillay realizaron esfuerzos y acercamientos con el movimiento LGBTI. Navi Pillay por pedido del Consejo de Derechos Humanos presento en 2011 el informe "Leyes y prácticas discriminatorias y actos de violencia cometidos contra personas por su orientación sexual e identidad de género" el cual documenta violaciones a los derechos humanos de personas LGTBI. El informe luego se transformó en la campana "Libres e Iguales" en la cual la OACDH se avoca a promover cinco obligaciones básicas que deben cumplir los Estados miembros de la ONU, estas son:

A) Proteger a las personas contra la violencia homofóbica y transfóbica;

B) Prevenir la tortura y los tratos crueles, inhumanos y degradantes contra las personas LGBTI que estén detenidas, prohibiendo y sancionando este tipo de actos y asegurando que se ofrezca una reparación a las víctimas; C) Derogar las leyes que tipifican penalmente la homosexualidad; D) Prohibir la discriminación basada en la orientación sexual y la identidad de género; E) Salvaguardar la libertad de expresión, de asociación y de reunión pacífica de las personas LGBTI e intersexuales (OACDH, 2012, p. 11).

${ }^{22}$ Arbour promovió la redacción de los principios de Yogyakarta. 
En 2014 los Estados árabes lograron elegir al jordano Zeid Ra’ad Al Hussein como nuevo Alto Comisionado, dicha victoria del mundo árabe no mudo el compromiso de la OACDH sobre la lucha contra la discriminación por OSIG. De hecho en su primer discurso ante el Consejo de Derechos Humanos el señor Zeid Ra'ad Al Hussein declaro que no hay justificación alguna, por la degradación o la explotación de otros seres humanos por cualquier motivo, incluyendo la orientación sexual (OHCHR, 2014). Lo anterior significaba su público compromiso por seguir el trabajo iniciado por su antecesora. Esto fue aún más claro al presentar el 4 de mayo de 2015, la actualización del informe solicitado por el Consejo de Derechos Humanos. En dicho informe la $\mathrm{OACDH}$ presento avances significativos en decenas de países que aprobaron leyes contra la discriminación y ampliaron la protección a las personas LGBTI. Sin embargo también presento un patrón universal de violaciones sistemáticas a los derechos humanos de las personas LGBTI. El informe suma nuevas recomendaciones al anterior, entre ellas esta promover legislación anti homofóbica y Transfobia, así como legislación que prohíba la incitación al odio homofóbico, también prohibir las terapias de conversión y cualquier tratamiento involuntario como esterilización forzada, mutilación genital o exámenes anales. El informe también incluye las violaciones a los derechos humanos de las personas intersexuales (OACDH, 2015).

Tanto Ban Ki-Moon como Zeid Ra'ad Al Hussein y sus antecesoras se han basado en defender el argumento de que no son nuevos derechos sino los mismos ya estipulados, así como se han enfocado en la lucha contra la descriminalización de la sodomía y la eliminación de actos crueles y violentos, siendo ambas preocupaciones que gozan de un mayor apoyo en la comunidad internacional, que otras reivindicaciones sobre discriminación. A la vez el acercamiento de ambas autoridades ha sido de comprensión, cooperación y horizontalidad con las naciones, evitando así confrontaciones con Estados sensibles al tema cuando es señalado por otro país de manera vertical. 


\subsection{Agencias especializadas y Programas de la ONU}

El 17 de mayo de cada año se celebra el Día Internacional contra la Homofobia y la Transfobia para recordar cuando la asamblea general de la OMS en 1990 eliminó la homosexualidad de la Clasificación Internacional de Enfermedades, la homosexualidad es vista hoy como una variación natural de la sexualidad humana y no una condición patológica. Otros avances de la OMS ha sido su condena las terapias de cambio de orientación sexual, reforzar el acceso a la salud de las personas LGTBI, la posibilidad de alcanzar un consenso que declare la transexualidad como otra variación natural de la sexualidad.

Otro importante avance ha sido que el Alto Comisionado de las Naciones Unidas para los Refugiados (ACNUR) ha incluido a las personas LGBTI como un grupo social pasible de ser considerado en solicitudes de asilo (ACNUR, 2012), reconociendo que este grupo califica plenamente en la definición de refugiado de la Convencion de Ginebra de 1951.

En septiembre de 2015 por primera vez 12 agencias de las Naciones Unidas hicieron una declaración en conjunto para frenar la discriminación y violencia contra las personas LGTBI, recordando el deber de los estados de protegerlas como manda el derecho internacional (ORGANIZAÇÃO..., 2015).

\section{POSICIONAMIENTO DE LOS MECANISMOS DE TRATADOS Y PROCEDIMIENTOS ESPECIALES}

Los procedimientos especiales han documentado y presentado en sus informes violaciones a los derechos humanos de las personas LGTBI, su trabajo ha llamado la atención de los estados en las visitas in loco y en sus presentaciones en la Asamblea General, el Consejo de Derechos Humanos y otros foros. Sin embargo hace falta evaluar el impacto que sus actuaciones han tenido a nivel local. Por su parte los comités de tratados 
han realizado una labor de monitoreo y promoción de los derechos humanos de las personas LGTBI. El Comité de Derechos Humanos ha sido el más activo al respecto.

En 1980 en el caso Hertzberg vs Finlandia el comité se convirtió en el primer espacio de la ONU que se discutía sobre la orientación sexual, sin embargo la decisión del comité en ese momento fue lamentable y homofóbica. El caso planteaba que Hertzberg había sido censurado por el gobierno de Finlandia al hablar sobre homosexualidad en un programa de televisión, el comité decidió que las medidas tomadas por Finlandia no podían ser cuestionadas por que la televisión no era un foro apropiado para hablar sobre la homosexualidad ya que podría tener efectos nocivos sobre los menores de edad que estuvieran asistiendo el programa (CCPR, 1980). Sin embargo 14 años más tarde en 1994 en el caso Toonen vs Australia, en dicho caso el comité concluyo que la ley anti sodomía era una injerencia en la vida privada de las personas, así como también incluyo la orientación sexual como una categoría sospechosa de discriminación dentro artículo 2 y 26 del pacto ${ }^{23}$ cuando hacen referencia al sexo (CCPR, 1994). El Comité reforzó esto en el caso X vs Colombia al dictaminar que "La prohibición contra la discriminación bajo el artículo 26 del Pacto Internacional de Derechos Civiles y Políticos incluye también la discriminación basada en la orientación sexual” (CCPR, 2007). Sin embargo en el caso Juliet Joslin y otras vs Nueva Zelanda donde las supuestas víctimas alegan que el Estado no les permite casarse como pareja lesbiana, el comité decidió que Nueva Zelanda no había violado el Pacto, por lo tanto desestimo la denuncia, el Comité dijo que el Pacto solo reconoce como matrimonio únicamente la unión entre un hombre y una mujer que desean casarse (CCPR, 2002). Con dicho dictamen el comité cerró la posibilidad de conseguir la obligación de que los estados no impidan el registro civil de uniones de personas

${ }^{23}$ Pacto Internacional de derechos civiles y políticos. 
del mismo sexo. Sin embargo en el caso Young vs. Australia donde la victima acuso al Estado de negarle una pensión de viudez al fallecer su pareja quien era veterano de guerra. Australia al no reconocer las uniones del mismo sexo se basó en ese criterio para negar dicho derecho. Además la ley australiana daba el beneficio a parejas casadas y no casadas heterosexuales, por lo tanto el comité dictamino que el Estado no había proporcionado argumentos que cumplan con los criterios de razonabilidad y objetividad, ${ }^{24} \mathrm{y}$ ninguna prueba que podría indicar la existencia de factores que justifican la distinción. Por lo que Australia había violado el artículo 26 del Pacto al denegar al autor una pensión en razón de su sexo u orientación sexual. (CCPR, 2003). El caso X vs. Colombia de 2007 fue resuelto con los mismos argumentos, reconociendo así que la discriminación por orientación sexual está prohibida según el pacto. En 2010 el comité decidió un caso similar al de censura de Hertzberg, donde la activista lésbica Irina Fedotova fue censurada y castigada por autoridades Rusia, luego de manifestarse cerca de una escuela. El comité en esta oportunidad decidió a favor del derecho de la activista a expresar su identidad sexual sin censura (CCPR, 2012). El comité también reconoció en el caso M. I. vs. Suecia (CCPR, 2013) el derecho a solicitar refugio a personas con temores fundados de violencia al volver a un país que criminaliza a las personas LGBTI. Las decisiones del comité de derechos humanos han causado un impacto positivo en el Sistema de Naciones Unidas en su conjunto y han servido como jurisprudencia para los sistemas nacionales de justicia.

Otros comités de tratados han abordado la no discriminación por orientación sexual, como el Comité de los Derechos de Niños y Niñas, en su comentario general número 4 de 2003, el Comité contra la Tortura, en su comentario general número 2 de 2008, el Comité para la Eliminación de la Discriminación contra la Mujer, en su recomendación general número

${ }^{24} \mathrm{El}$ comité concluyo que el argumento utilizado por Australia de que dicha ley protegía la moral pública, no carecía objetividad suficiente para limitar el derecho a la privacidad. 
28 de 2010 y el Comité de Derechos Económicos, Sociales y Culturales, en su comentario general número 20 de 2009. También los comités de tratados han introducido en los exámenes periódicos a los Estados partes recomendaciones y llamados de atención por violaciones a los derechos humanos de las personas LGBTI. Ha sido a través de los mecanismos de tratados que avances sobre la no discriminación y violencia por OSIG en el derecho internacional de los derechos humanos ha sido logrado en la ONU.

\section{CONCLUSIONES}

Este artículo se plantea al inicio dar respuestas a las siguientes interrogantes; ¿Cuáles son los principales argumentos que definen el debate sobre la Orientación Sexual e Identidad de Género en la ONU? Respecto a esta cuestión, podemos concluir que existe una confrontación entre la interpretación del principio de universalidad de los derechos humanos, los límites de la aplicación de la soberanía y la responsabilidad de los Estados de proteger, promover y respetar los derechos de todas las personas, incluidas las personas LGBTI. Los Estados que se oponen al reconocimiento de los derechos de las personas LGBTI, mantienen argumentos cargados de prejuicio y estereotipos que generan intolerancia, violencia y discriminación. Sin embargo cada vez más países de todos los continentes se suman al reconocimiento internacional de los derechos humanos de las personas LGBTI e importantes avances se han materializado en la ONU en los últimos 10 años. Las resoluciones del Consejo de Derecho Humanos, la reciente creación del nuevo experto sobre OSIG y los avances alcanzados en el EPU son muestra de que se está logrando alcanzar un mayor consenso. Esto es importante porque son los Estados los principales actores en el derecho internacional. 
La otras dos cuestiones corresponden a ¿Cómo las Naciones Unidas se posicionan sobre la protección a las personas LGBTI?, y, ¿Cómo los mecanismos de protección internacional han interpretado los pactos y convenciones para proteger a las personas LGBTI? A lo que podemos responder afirmando que las Naciones Unidas, las autoridades y sus agencias han determinado y defienden que las personas LGTBI están protegidas por los instrumentos y mecanismos del derecho internacional de los derechos humanos.

Enfrentar y sobrepasar los desafíos actuales y futuros dependerá de la constante movilización de la sociedad civil hacia sus gobiernos, así como seguir esa fuerte convicción de la ONU y sus funcionarios por abrir las mentes y los corazones de todos los líderes y las sociedades del mundo.

\section{REFERENCIAS}

ACNUR. Directrices sobre Protección Internacional: Solicitudes de la condición de refugiado relacionadas con la orientación sexual y/o la identidad de género en el contexto del artículo $1 A$ (2) del Estatuto de los Refugiados de 1951 y/o su Protocolo 1967. México, 2012.

ALVES, J. A. L. Os direitos humanos como tema global. Sao Paulo: Perspectivas, 2003. $41 \mathrm{p}$.

ARC INTERNATIONAL. Arc-international.net. 2005 Joint Statement, 2005. Disponivel em: $<$ http://arc-international.net/global-advocacy/sogi-statements/2005-joint-statement/>.

CCPR. Leo R-Hertzberg, Uit Mansson, Astrid Nikula and Marko and Tuovi Putkonen, represented by SETA (Organization for Sexual Equality) v. Finland, Communication No. R.14/61, U.N. Doc. Supp. No. 40 (A/37/40) at 161 (1982). Ginebra, 1980.

Nicholas Toonen v. Australia. Ginebra, 1994. 
CCPR. Juliet Joslin y otras v. New Zealand. Comite de Derechos Humanos. Ginebra, 2002.

. R. Edward Young v. Australia. Ginebra, 2003.

.X v. Colombia. Ginebra, 2007.

. Irina Fedotova v. Russian Federation, Communication No. 1932/2010, U.N. Doc. CCPR/C/106/D/1932/2010 (2012). Ginebra, 2012.

. M. I. v. Sweden, Communication No. 2149/2012, U.N. Doc. CCPR/ C/108/D/2149/2012 (2013). Ginebra, 2013.

CONSEJO DE DERECHOS HUMANOS. CDH. Resolución aprobada por el Consejo de Derechos Humanos; Derechos humanos, orientación sexual e identidad de género. Ginebra, 2011.

. Resolución aprobada por el Consejo de Derechos Humanos; Derechos humanos, orientación sexual e identidad de género. Ginebra, 2014.

EDWARD H. LAWSON, M. L. B. Encyclopedia of Human Rights. [S.1.]: Taylor \& Francis, 1996.

GIRARD, F. Negotiating Sexual Rights and Sexual Orientation at the UN/ SexPolitics - Reports from the Front Lines. [S.1.]: Sexpolitics, 2004.

INTERNATIONAL LESBIAN AND GAY ASSOCIATION. Ilga. The resolution on Human rights and sexual orientation. 2003. Disponivel em: $<\mathrm{http}: / / \mathrm{old}$. ilga.org/news_results.asp?LanguageID $=1 \&$ FileCategory $=44 \&$ FileID $=406>$. . Homofobia de Estado 2016: Un estudio mundial jurídico sobre la criminalización, la protección y el reconocimiento del amor entre personas del mismo sexo. Ginebra, 2016.

JESUS, B. D. La Resolución Brasileña en la ONU y la movilización para su aprobación, 7 abr. 2004. Disponivel em: <http://equidadecuador.org/es/todo/ ATT1111618449-1.pdf>.

KI-MOON, B. La promesa de Ban Ki-Moon. unfe.org, 2015. Disponivel em: $<$ https://www.unfe.org/es/actions/ban-ki-moon-oslo>. 
NACIONES UNIDAS. Carta de las Naciones Unidas. San Francisco: [s.n.], 1945.

OACDH. Nacidos Libres e Iguales; Orientación sexual e identidad de género en las normas internacionales de derechos humanos. Ginebra, 2012. Report of the Office of the United Nations High Commissioner for Human Rights "Discrimination and violence against individuals based on their sexual orientation and gender identity”. Ginebra, 2015.

OHCHR. Opening Statement by Zeid Ra'ad Al Hussein United Nations High Commissioner for Human Rights at the Human Rights Council 27th Session, 8 Septiembre 2014. Disponível em: <http://www.ohchr.org/EN/NewsEvents/ Pages/DisplayNews.aspx?NewsID=14998\&LangID=E $>$.

ORGANIZAÇÃO DAS NAÇÕES UNIDAS. ONU. World Conference against Racism, Draft Program. Durban, 2001.

. In Series of Votes, SOCIAl Committee Defeats Efforts to Amend Texts on Extrajudicial, Summary or Arbitrary Executions, 22 nov. 2002. Disponível em: <http://www.un.org/press/en/2002/GASHC3730.doc.htm>.

Commission on Human Rights Approves Measures on Promotion and Protection OF Human Rights, Other Issues, 2003a. Disponivel em: <http://www. un.org/press/en/2003/hrcn1045.doc.htm>.

. UN News - After gay rights event, Annan calls for tolerance, laments bias 'on any grounds, 5 ago. 2003b. Disponível em: <http://www.un.org/apps/ news/story.asp?NewsID $=7917 \& \mathrm{Cr}=$ gay $\& \mathrm{Cr} 1=$ rights $>$.

. Consejo de Derechos Humanos, Enmienda Proyecto de Resolución Protección de la familia, 2014a. Disponível em: <http://ap.ohchr.org/Documents/S/HRC/d_res_dec/A_HRC_26_L37.pdf>.

. Consejo de Derechos Humanos, Proyecto de Resolución Protección de la familia, 2014b. Disponível em: <http://ap.ohchr.org/Documents/S/HRC/d_ res_dec/A_HRC_26_L20.pdfs. 
ORGANIZAÇÃO DAS NAÇÕES UNIDAS. ONU. Prohibition of discrimination, harassment, including sexual harassment, and abuse of authority, 11 feb. 2008. Disponível em: <http://daccess-dds-ny.un.org/doc/UNDOC/GEN/ N08/238/36/PDF/N0823836.pdf?OpenElement>.

Twelve UN agencies issue unprecedented joint statement on rights of lesbian, gay, bisexual, transgender \& intersex people, 2015. Disponível em: $<$ http://www.ohchr.org/EN/NewsEvents/Pages/DisplayNews.aspx?NewsI$\mathrm{D}=16511 \&$ LangID=E\#sthash.sG57cyIm.dpuf .

Protection against violence and discrimination based on sexual orientation and gender identity, 30 jun. 2016. Disponivel em: <http://www.ohchr. org/EN/NewsEvents/Pages/DisplayNews.aspx?NewsID=20220\#sthash. dY4IwMK7.dpuf>.

PERMANENT MISSION OF SYRIA. ONU. [S.1.]. 2008.

REUTERS. Russia fails in bid to stop U.N. staff benefits for all gay couples, 24 mar. 2015. Disponível em: <http://www.reuters.com/article/us-un-gaymarriage-russia-idUSKBN0MK1UW20150324\#TVHPFmE83H2Cxerr.99>.

RODRIGUES, Gilberto M. A. Organizacoes internacionais. Sao Paulo: Moderna, 2014.

SANDERS, D. Human Rights and Sexual Orientation in International Law. International Journal of Public Administration, 2002.

SEYCHELLES NEWS AGENCY. Seychelles parliament passes bill to decriminalize sodomy, Victoria, 18 May 2016.

VATICANO. Vatican.va. Intervención en la 63 sesión de la Asamblea general de la ONU, 18 Diciembre 2008. Disponível em: <http://www.vatican.va/roman_ curia/secretariat_state/2008/documents/rc_seg-st_20081218_statement-sexual-orientation_sp.html>.

Recebido em: 7/11/2016

Aceito em: 18/12/2016 